\title{
¿QUÉ APORTA LA PERSPECTIVA DE GÉNERO AL URBANISMO?
}

\author{
Zaida MuXí Martínez, Roser Casanovas, Adriana \\ Ciocoletto, Marta Fonseca y Blanca GutiÉrRez VAldivia \\ Universidad Politécnica de Cataluña
}

Recibido 09/02/2011

Aceptado 20/05/2011

\section{Resumen}

¿Qué significa un urbanismo con perspectiva de género? ¿Cómo hacer planificación urbanística y proyectos urbanos que tengan en cuenta la perspectiva de género? El objetivo del urbanismo deberia ser poder vivir en barrios inclusivos que tengan en cuenta la diversidad real que caracteriza a los espacios urbanos, y así hacer posible que el derecho a la ciudad sea un derecho humano para todas las personas. Pensar el espacio urbano para todas y para todos es hacerlo desde la diferencia pero no desde la desigualdad: una diferencia entre mujeres y hombres, entre clases, entre orígenes, culturas, religiones, etc. posicionando en igualdad de condiciones todas las demandas, sin decisiones a priori, que excluyan la experiencia de las personas como fuente fundamental de conocimiento en las deciciones urbanas.

Palabras claves: Planificación urbanística. Proyecto urbano. Perspectiva de género. Participación urbana. 


\begin{abstract}
What is the meaning of gender urban perspective? How can we make urban planning and urban projects that have gender mainstreaming? The main objective of urban planning should be the fact of being able to enjoy inclusive cities that take into account the diverse population that inhabits them, thus making the city a human right for all. To think about urban space for all is to do it from the difference between people but not from the inequality, the difference between women and men, between classes, origins, culture, religions etc, placing in equal condition all the demands without excluding the experiences of people and without taking any decision by ourselves, consider this experiences as the fundamental source for urban planning decisions.
\end{abstract}

Keywords: Urban Panning. Urban project. Gender perspective. Urban participation. 
¿Qué significa un urbanismo con perspectiva de género? ¿Cómo hacer planificación urbanística y proyectos urbanos que tengan en cuenta la perspectiva de género? Habitar es mucho más que la sumatoria de la residencia, el trabajo, las tareas del hogar, el ocio, el transporte, la educación, la cultura, los deportes y la sanidad. Habitar es poder desarrollar las diferentes esferas de la vida en igualdad de oportunidades, con intensidad e integridad. Por ello consideramos necesario pensar y, sobre todo, repensar las ciudades y los barrios guiados por esta idea. Poner en primer plano la vida y las necesidades de las personas es una tarea compleja, no exenta de complicaciones.

Por lo tanto, ¿qué significa repensar un barrio con perspectiva de género? ¿Significa trabajarla sólo para las mujeres, es decir, en oposición a la de los hombres? No; se trata de pensar un barrio y una ciudad con todos sus detalles y a través de todas las escalas desde la complejidad y la diversidad, sin dar prioridades exclusivas a consideraciones económicas alejadas de las personas. Se trata de construir, o reconstruir, barrios que no perpetúen las diferencias y las desigualdades de género, clase, raza o edad. Se trata de ponerse las gafas lila ${ }^{1}$ y volver a estudiar, analizar y registrar la realidad para conseguir entornos urbanos más adecuados.

El objetivo del urbanismo deberia ser poder disfrutar de ciudades inclusivas que tengan en cuenta la diversidad real que caracteriza a los espacios urbanos, y así poder hacer posible que el derecho a la ciudad sea un derecho humano para todas las personas. ¿Qué quiere decir hoy el derecho a la ciudad? ¿Es todavía vigente denominar de manera universal los sujetos de derecho? ¿Puede existir el sujeto universal como homogeneizador de las necesidades, derechos y voluntades? ¿Es aceptable que el sujeto universal, enmascarado en el masculino, englobe de manera genérica hombres y mujeres? Los espacios físicos condicionan el derecho a la ciudad, entendida según el artículo I de la Carta Europea de Salvaguarda de los Derechos Humanos en la Ciudad como espacio colectivo que pertenece a todos los habitantes, los cuales tienen derecho a encontrar las condiciones para su realización política, social y ecológica, asumiendo deberes de solidaridad.

1. LiENAS, Gemma; El diari lila de la Carlota. Barcelona: Editorial Empuries, 2001.

Feminismo/s 17, junio 2011, pp. 105-129 
La universalidad esconde el sujeto real de derecho, que es quien ha conformado con sus necesidades la falsa neutralidad. Según Cristina Carrasco ${ }^{2}$ el universalismo es una forma de enmascarar que el sujeto de los derechos de ciudadanía es masculino. El problema de fondo es que la ciudadanía, y por lo tanto el derecho a la ciudad y las prioridades en la definición de esta, se ha construido tomando como referencia el mundo público, la participación en el mercado y los espacios asignados a los hombres. El espacio doméstico-femenino no está incluido en la categoría de ciudadanía. Pero, como dice Anna Bofill:

La gestión de la vida cotidiana no se produce solamente en el interior de las paredes que conforman la vivienda. Es también un conjunto de actividades, comportamientos, sensaciones y experiencias que se producen en el espacio público, en el espacio hasta ahora considerado masculino. ${ }^{3}$

Hay que reconocer la importante aportación a la economía y la sociedad de las tareas de atención y cuidados, tanto físicos como psíquicos, tradicionalmente asignadas a las mujeres, contribuciones que no han sido reconocidas. El urbanismo y la planificación tienen que considerar las necesidades derivadas de estas tareas como puntos imprescindibles para diseñar una ciudad inclusiva.

Hay que prescindir de falsas mitificaciones que quieren construir un pasado idílico de personas iguales y asumir la complejidad de la realidad que vivimos a comienzos del siglo XXI. Como dice Saskia Sassen, la ciudadanía no siempre aporta cumplidos e iguales derechos a todas las personas. A pesar del reconocimiento formal de la igualdad, miembros de grupos oprimidos por razones de etnia, religión, género u orientación sexual se enfrentan de forma cotidiana a diferentes formas de exclusión que los imposibilitan de la plena participación en la vida pública.

\section{Aplicar la perspectiva de género en el urbanismo}

La perspectiva de género aplicada al urbanismo significa poner en igualdad de condiciones las exigencias derivadas del mundo productivo y las derivadas del mundo reproductivo, es decir, las necesidades cotidianas de atención a las personas. Los dos mundos tienen que situarse en el mismo nivel de importancia en las decisiones para evitar ampliar o mantener las diferencias.

2. Carrasco, Cristina i Serrano, Mònica; Compte satèl $\bullet$ lit de la producció domèstica(CSPD) de les llars de Catalunya 2001, col•lecció Estudi 1, Institut Català de les Dones, Generalitat de Catalunya, octubre del 2006.

3. Bofill LeVI, Anna; Planejament urbanístic, espais urbans i espais interiors des de la perspectiva de les dones. Quaderns de l'Institut / 6. Barcelona, Generalitat de Catalunya, Institut Catalá de les Dones, Departament de Política Territorial i Obres Públiques, 2005, p. 30. 
Cuando analizamos la ciudad como un todo, en una escala lejana, obtenemos datos de distribución de los equipamientos, de transportes, de conectividad y accesibilidad, así como las posibilidades de relación entre áreas, etc. Estos datos siempre se tienen que confirmar desde la proximidad. La cuantificación numérica y las proporciones según las necesidades de equipamientos y áreas verdes, son un primer peldaño en la planificación y la organización territorial. Aun así, una vez hemos cumplido y demostrado que numéricamente se resuelven todas las necesidades, tenemos que acercarnos y entender cada uno de estos elementos dentro del funcionamiento de las redes cotidianas. La creación de sectores de suelo, de polígonos no cualificados que esperan la construcción de los equipamientos u otros servicios no son mecanismos suficientes para garantizar el desarrollo de la vida cotidiana de las personas.

Los equipamientos se tienen que entretejer con los recorridos de cada día, es decir no se tienen que colocar en los límites de las áreas urbanizadas puesto que genereran calles sin vida y con falta de seguridad. Con la expulsión de los equipamientos a las afueras de nuestras ciudades perdemos uno de las máximas ventajas de la mezcla y la proximidad: conseguir que los recorridos representen tiempos útiles. Es decir, poder utilizar el tiempo eficientemente si los diversos itinerarios cotidianos nos permiten satisfacer las necesidades cotidianas. En cambio, el uso del tiempo no es eficiente cuando cada recorrido es exclusivo para una función.

En la ciudad lo mejor que puede pasar es la «promiscuidad» de funciones, es decir, hacer una planificación que nos permita la mezcla de usos, no sólo en parcelas contiguas sino en las mismas parcelas. La ciudad tiene que ser cono una cebolla con miles de hojas iguales y diferentes a la vez: iguales en relevancia e impresindibilidad y diferentes en los usos, en los usuarios y usuarias y en los tiempos de utilización, de este modo garantizaremos, como diria Jane Jacobs $^{4}$ una danza urbana constante.

La perspectiva de género aplicada al urbanismo se entiende desde la experiencia y, por lo tanto, la escala próxima, pero esto no excluye la lectura analítica de la estructura general, que nos permitirá hacer recomendaciones en otros momentos de la planificación, más generales y lejanos, como puede ser un plan general.

No tenemos que olvidar que muchas decisiones quedan hipotecadas en esta etapa de la planificación donde las personas son, en el mejor de los casos, datos estadísticos incompletos porque no tenemos suficientes datos

4. JACOBS, Jane; Muerte y Vida de las grandes ciudades. Barcelona: Ed.62, 1967.

Feminismo/s 17, junio 2011, pp. 105-129 
segregados o porque estas se basan en áreas censales que distorsionan la distribución territorial de las personas y, por lo tanto, de las necesidades.

La planificación urbana tendría que enfocarse de forma que nos permita hacer un constante trabajo transversal, transescalar e interdisciplinario. Transversal entre las personas profesionales que trabajan y entre las áreas del mismo ayuntamiento, otras instituciones y también poblaciones implicadas, es decir, transversalidad vertical y horizontal.

Transescalar significa trabajar verificando las propuestas siempre desde la proximidad. Los departamentos de urbanismo y vivienda deciden las formas, esto significa un gran poder y una gran responsabilidad; a pesar de esto hay que tener en cuenta que el conocimiento urbano es compartido pero diferente en las diferentes áreas de trabajo y conocimiento.

En la sociedad actual, los roles de género tienden a difuminarse y las tareas asignadas tradicionalmente a las mujeres dejan de ser exclusividad suya. Sin embargo, en el proyecto urbano, las decisiones sobre las viviendas y los equipamientos (horarios, características, localización, etc.) se siguen pensando desde la división de roles, como si existiera una persona que tuviera un horario liberado para dedicarlo a la atención a las persones dependientes o para el cuidado del hogar.

La Ley orgánica 3/2007, llamada ley de igualdad, ha sido redactada para hacer posible el derecho a la igualdad real entre mujeres y hombres, el cual ya se proclama en el artículo 14 de la Constitución española y es un principio jurídico universal reconocido en varios textos internacionales sobre derechos humanos, y así se recoge en el preámbulo de dicha ley:

El pleno reconocimiento de la igualdad formal ante la ley, a pesar de haber comportado, sin duda, un paso decisivo, ha resultado insuficiente. La violencia de género, la discriminación salarial, la discriminación en las pensiones de viudedad, el hecho que haya más desocupación femenina, la todavía escasa presencia de las mujeres en lugares de responsabilidad política, social, cultural y económica, o los problemas de conciliación entre la vida personal, laboral y familiar, demuestran que la igualdad plena, efectiva, entre mujeres y hombres, la «perfecta igualdad que no admite poder ni privilegio para unos ni incapacitadada para otros», en palabras escritas por John Stuart Mijo hace casi 140 años, hoy todavía es una tarea pendiente que necesita nuevos instrumentos jurídicos.

Como la Ley busca la prevención, entiende que la construcción del medio que habitamos no es neutral, por eso hace referencia al impacto de género de diferentes actuaciones de relevancia económica, social, cultural y artística. Con anterioridad a esta Ley, en las disposiciones adicionales del Decreto legislativo 1/2005, de 26 de julio, que aprueba el Texto refundido de la ley de 
urbanismo ${ }^{5}$ la disposición decimocuarta lleva como título «Incorporación de la perspectiva de género», y dice:

El Departamento de Política territorial y obras públicas tiene que incorporar la perspectiva de género en el desarrollo de esta Ley para garantizar la promoción de la representación paritaria en la composición de los órganos urbanísticos colegiados y de la evaluación del impacto de la acción urbanística en función del género.

La ley de igualdad también determina en su articulado una serie de cuestiones que pueden ser de gran utilidad para confirmar la pertinencia de llevar a cabo un urbanismo que incorpore la perspectiva de género como herramienta esencial. Lo encontramos en el artículo 20, Adecuación de las estadísticas y estudios; en artículo 22, Acciones de planificación equitativa de los tiempos y, especialmente, el artículo 31, Políticas urbanas, de ordenación territorial y vivienda. En resumen:

1. Las políticas y los planes de las administraciones públicas en materia de acceso a la vivienda tienen que incluir medidas destinadas a hacer efectivo el principio de igualdad entre mujeres y hombres. Del mismo modo, las políticas urbanas y de ordenación del territorio tienen que tener en consideración las necesidades de los diferentes grupos sociales y de los varios tipos de estructuras familiares, y favorecer el acceso en condiciones de igualdad a los diferentes servicios e infraestructuras urbanas.

2. El Gobierno, en el ámbito de sus competencias, tiene que fomentar el acceso a la vivienda de las mujeres en situación de necesidad o en riesgo de exclusión, y de las que hayan sido víctimas de la violencia de género, especialmente cuando, en los dos casos, tengan hijos menores exclusivamente a su cargo.

3. Las administraciones públicas tienen que tener en cuenta en el diseño de la ciudad, en las políticas urbanas, en la definición y ejecución del planeamiento urbanístico, la perspectiva de género, utilizando para hacerlo, especialmente, mecanismos e instrumentos que fomenten y favorezcan la participación ciudadana y la transparencia.

\section{Recomendaciones para la aplicación de la perspectiva de género}

Las recomendaciones se basan en la convicción de la necesaria incorporación de la perspectiva de género en el conocimiento de los mecanismos de la planificación y los proyectos de urbanización así como en la observación de las características físicas y sociales para lograr el cumplimiento efectivo de la Ley orgánica 3/2007 según la cual:

5. Texto refundido de la Ley de Urbanismo y de su Reglamento aprobado por el Decreto 305/2006, de 18 de julio. 
La prevención de estas conductas discriminatorias y en la previsión de políticas activas para hacer efectivo el principio de igualdad. Esta opción implica necesariamente una proyección del principio de igualdad sobre los diversos ámbitos del ordenamiento de la realidad social, cultural y artística en que se pueda generar o perpetuar la desigualdad. De aquí la consideración de la dimensión transversal de la igualdad, señal de identidad del moderno derecho antidiscriminatorio, como principio fundamental de este texto.

La Ley se refiere a la generalidad de las políticas públicas en España, tanto estatales como autonómicas y locales:

Artículo 15. Transversalidad del principio de igualdad de trato entre mujeres y homes.el principio de igualdad de trato y oportunidades entre mujeres y hombres tiene que informar,con carácter transversal, la actuación de todos los poderes públicos. Las administraciones públicas lo tienen que integrar, de manera activa, en la adopción y ejecución de las disposiciones normativas, en la definición y presupuestos de políticas públicas en todos los ámbitos y en el desarrollo del conjunto de todas las actividades.

\subsection{Un análisis diferenciado e integrado}

La legislación urbanística divide la problemática del territorio según escalas, segregando problemas y necesidades que tendrían que plantearse en conjunto. Es imprescindible intervenir en esta división puesto que las relaciones y actividades cotidianas no se encuentran segregadas en la vida de las personas, especialmente en la vida de las mujeres, en quienes recae todavía de manera mayoritaria la responsabilidad de hacer encajar esferas y tiempos diferentes. Según la encuesta demogràfica del 2007, en Cataluña ${ }^{6}$ el 47\% de las mujeres asume la práctica totalidad de los trabajos no remunerados del hogar mientras que el porcentaje de hombres que se responsabilizan de casi todos los trabajos domésticos es sólo del 8,8\%. Los porcentajes de no participación en el cuidado de los hijos en el hogar son más altos en hombres $(23,9 \%)$ que en mujeres $(9,4 \%)$. La atención compartida de las criaturas es la situación más frecuente, de forma que en torno el 43,5\% de los hombres y de las mujeres dicen repartir el trabajo de cuidar los niños y niñas con al menos otra persona del hogar. Un 38,6\% de las mujeres asume en su práctica totalidad el cuidado de las criaturas, lo cual indica que conviven con personas que no participan o que tienen una mínima participación en los trabajos de atención a los pequeños de la casa, incluyendo también las situaciones de familias monoparentales, que representan el 19\% de los núcleos con hijos. En el 85\% de los núcleos monoparentales la persona progenitora presente es la madre.

6. <http://www.idescat.cat/cat/idescat/serveis/premsa/ed2007ds.pdf> Consultado el 07-02-2011. 
La segregación de decisiones según la escala de trabajo ha generado una falta de coherencia en muchos territorios que se manifiesta especialmente en los barrios y en sus entornos, en la falta de redes de proximidad para el desarrollo de la vida cotidiana.

Por eso, la aplicación de la perspectiva de género en los proyectos urbanos necesita como punto de partida una nueva forma de análisis, diferente a las empleadas habitualmente. Este análisis tiene que introducir de una forma exhaustiva criterios físicos y sociales en todas las escalas y fases del planeamiento de manera transversal. No se puede entender y estudiar un territorio o un entorno específico sin entender y estudiar las personas que viven, sus especificidades y sus necesidades diversas.

Aunque estudiamos el detalle no se pueden dejar de atender las consecuencias que repercuten en el entorno próximo y más amplio, y a la inversa, el planeamiento general tiene que atender a la particularidad de cada calle y de cada línea que se dibuja. Por lo tanto, es necesario un análisis diferenciado de cada circunstancia o problema a resolver en cada proyecto, pero es fundamental que estén integrados, es decir, que cada variable esté entretejida con el resto.

El cambio fundamental que propone la aplicación de la perspectiva de género en la construcción de las ciudades y los pueblos es priorizar los seres humanos concretos y sus necesidades en todos los niveles de planeamiento, teniendo como objetivo principal hacer barrios y ciudades con redes adecuadas para la vida cotidiana de todas las personas que conviven en un territorio.

\subsection{Variables de análisis de la realidad}

El entorno cotidiano es un complejo tejido de variables. Para poder facilitar el estudio de esta red las hemos dividido en seis ${ }^{7}$. Los espacios públicos o de relación, los equipamientos, la movilidad y la vivienda son las variables que definen el apoyo físico sobre el cual se desarrolla la red cotidiana. La participación y la seguridad son conceptos que influirán a su vez en la definición física. Las seis variables, no pueden entenderse si no como temas a trabajar necesariamente de manera transversal.

7. MuXí MARTINEZ, Zaida y COL.LECTIU PUNT 6; Recomanacions per la implementació de polítiques de gènere al urbanisme. Entidad financiadora: Institut Català de les Dones, expediente U-62/06. Convenio de colaboración con la Universitat Politècnica de Catalunya grupo de investigación I-00868. 2006-2007. <www.punt6.net> Consultado el 07-02-2011. 
Espacios públicos de relación

Los espacios de relación pensados para la equidad de género favorecen la autonomía y la socialización de las personas puesto que han sido pensados priorizando en su diseño y trazado las necesidades de mujeres y hombres.

Para conseguirlo son imprescindibles estos elementos: aceras anchas y activas que favorezcan la realización de diferentes actividades, con diferenciación de materiales, colores y texturas en los cambios de nivel; alcorques al mismo nivel de la acera; barandillas y rampas en las zonas con pendiente; pasos de peatones muy diferenciados y con semáforos que tengan en cuenta los tiempos lentos; bancos con sombras que generen zonas de descanso en los recorridos urbanos y en las áreas de estancia; espacios intermetdios de relación entre los interiores y exteriores; señalización que facilite la orientación, la identificación y la apropiación. Se tienen que trabajar los espacios de relación de manera inclusiva, pensando en todas las edades y condiciones de las personas que lo usarán, incorporando espacios específicamente pensados para grupos concretos de usuarios y usuarias.

El reconocimiento de la importante tarea de las mujeres en la sociedad tiene que hacerse visible en el espacio público y uno de los mecanismos más inmediatos para conseguirlo es denominarlos en femenino, es decir, dar a los

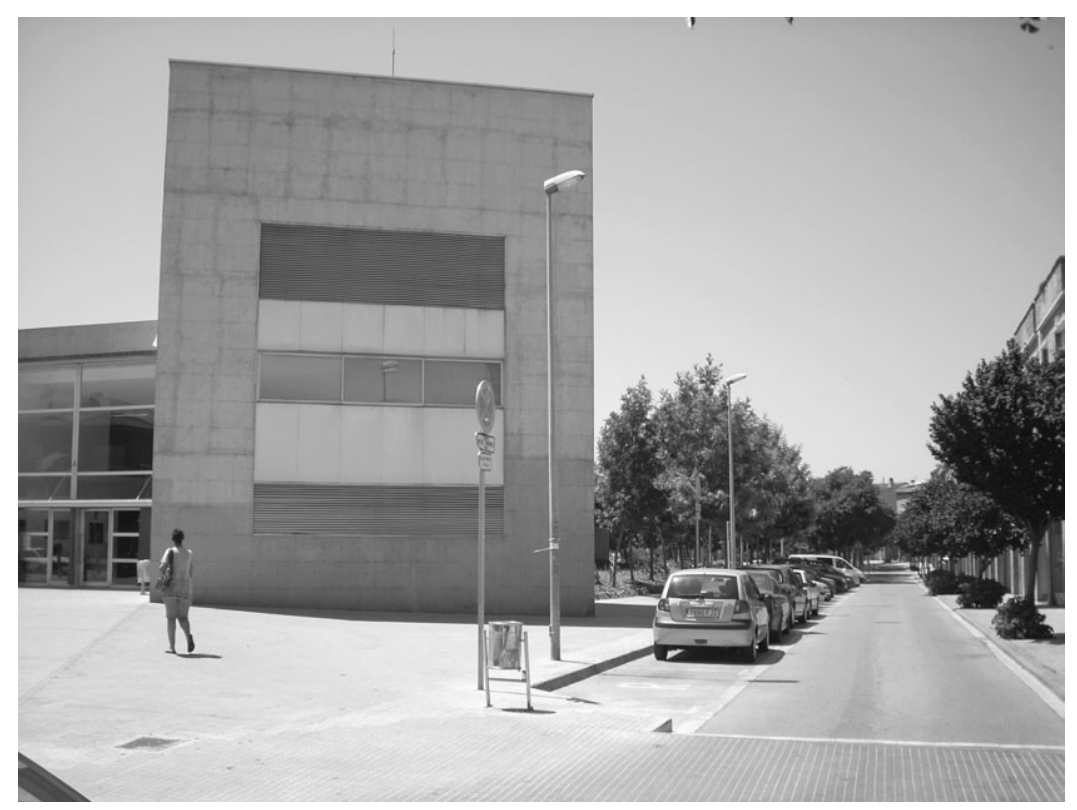

Feminismo/s 17, junio 2011, pp. 105-129 


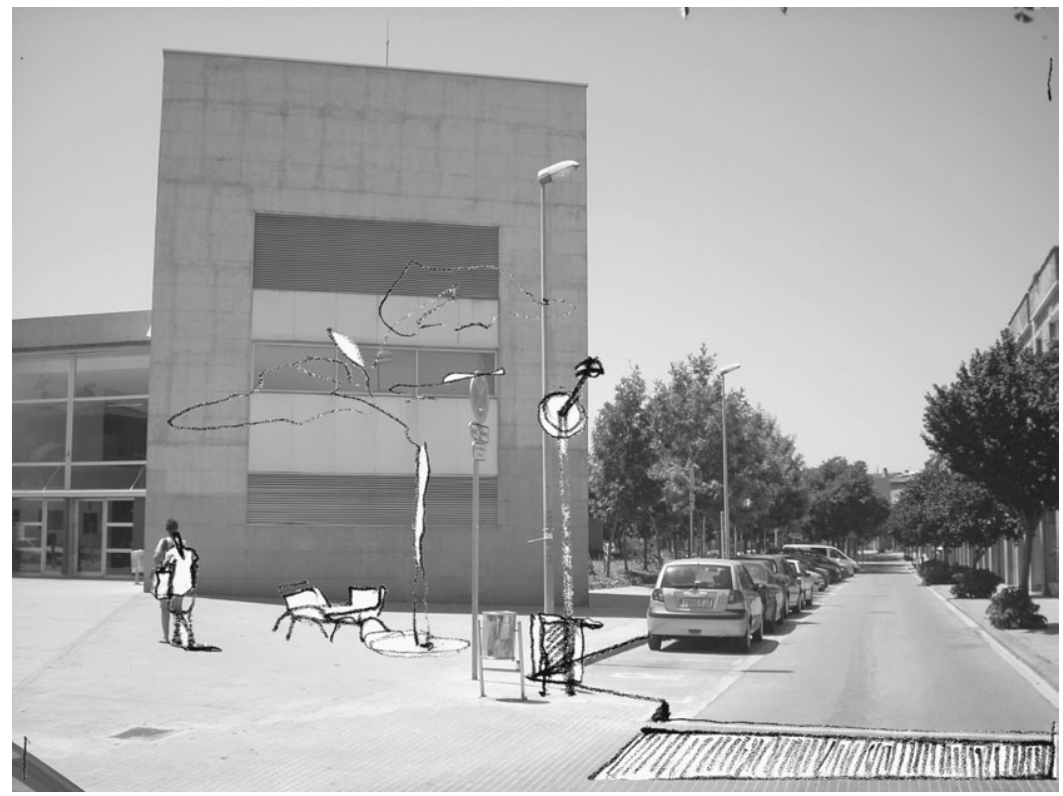

parques, plazas y calles nombres de mujeres y explicar cuál fue su aportación a la sociedad. Tal como señala el Manual práctico para una señalización urbana igualitaria ${ }^{8}$ de la Federación Española de Municipios y Provincias, se tienen que trabajar las señalizaciones urbanas para hacer visible la igualdad de hombres y mujeres en el derecho a la ciudad y a sus espacios públicos. La carencia de figuras femeninas en las señales es una dimensión más que nos revela que, bajo la apariencia de universalidad en la concepción de la ciudad, hay un orden androcéntrico que se reproduce con la falsa neutralidad.

\section{Equipamientos y servicios}

La oferta de equipamientos se amplia cuando la sociedad en la que se inscriben reconoce, asume y valora el trabajo derivado de los roles de género. El espacio de los equipamientos necesita entretejerse al espacio público de la ciudad con la máxima permeabilidad y flexibilizando su utilización para maximizar la utilización de los espacios. Su distribución en el territorio en relación a los usos y a las redes de movilidad garantiza la calidad de vida, porque

8. www.femp.es/.../Manual\%20práctico\%20para\%20una\%20señalización\%20igualitaria.pdf. Consultado el 09-02-2011. 

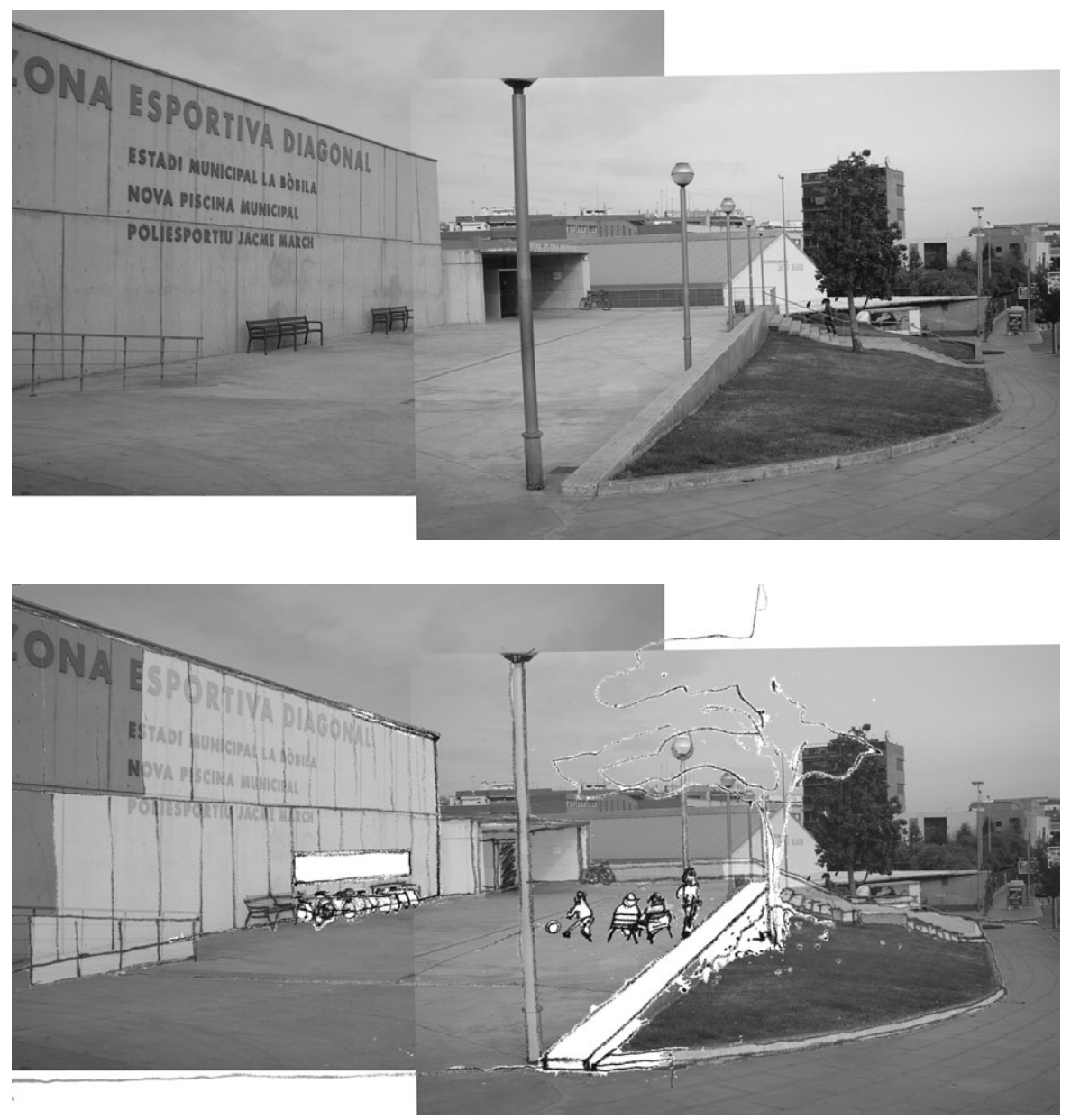

los convierte en estrategias para fomentar redes sociales, generar intercambio de servicios y posibilitar diferentes tiempos de uso acercando su funcionamiento a la complejidad de la vida cotidiana.

Movilidad

La oferta de medios de transporte y las características de los espacios soportes de la movilidad determinan formas y calidades de vida. La movilidad ha de ofrecer la máxima cantidad de variedad de opciones privilegiando los recorridos peatonales que se apoyan en un tejido urbano funcionalmente variado. 

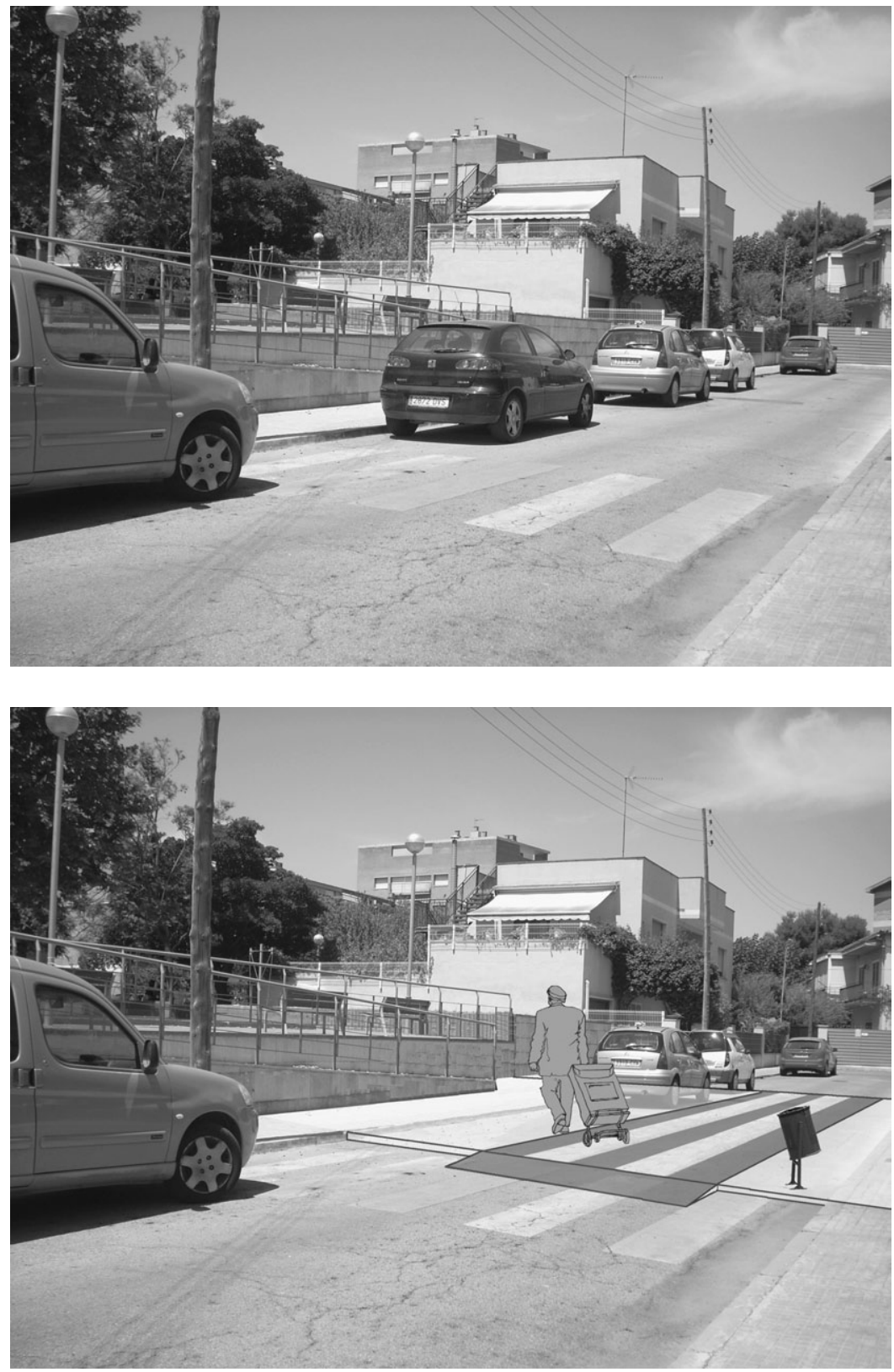

Los transportes públicos tienen que dar respuesta a la variedad de horarios del mundo reproductivo que genera recorridos no lineales ni uniformes. 
Se debe pensar la movilidad respondiendo a la diversidad y a la seguridad en todos sus aspectos, para facilitar la planificación de todas las actividades de la vida cotidiana y haciendo posible la igualdad de oportunidades en el acceso a la ciudad.

Vivienda

El espacio representa los valores sociales que lo han creado, por ello las viviendas han de reconocer las tareas de cuidado del hogar y las personas dándoles un espacio. Las tareas del hogar han de ser compartidas, no se deberían esconder no dejar la responsabilidad de las mismas en una sola persona.

Es necesario aprovechar los edificios de viviendas para generar espacios de relación entre vecinos, haciendo un uso compartido de servicios y construyendo espacios de tránsito entre lo privado y lo público. Es importante que en las plantas bajas se ofrezcan actividades variadas en relación directa con el espacio público favoreciendo la seguridad del mismo.

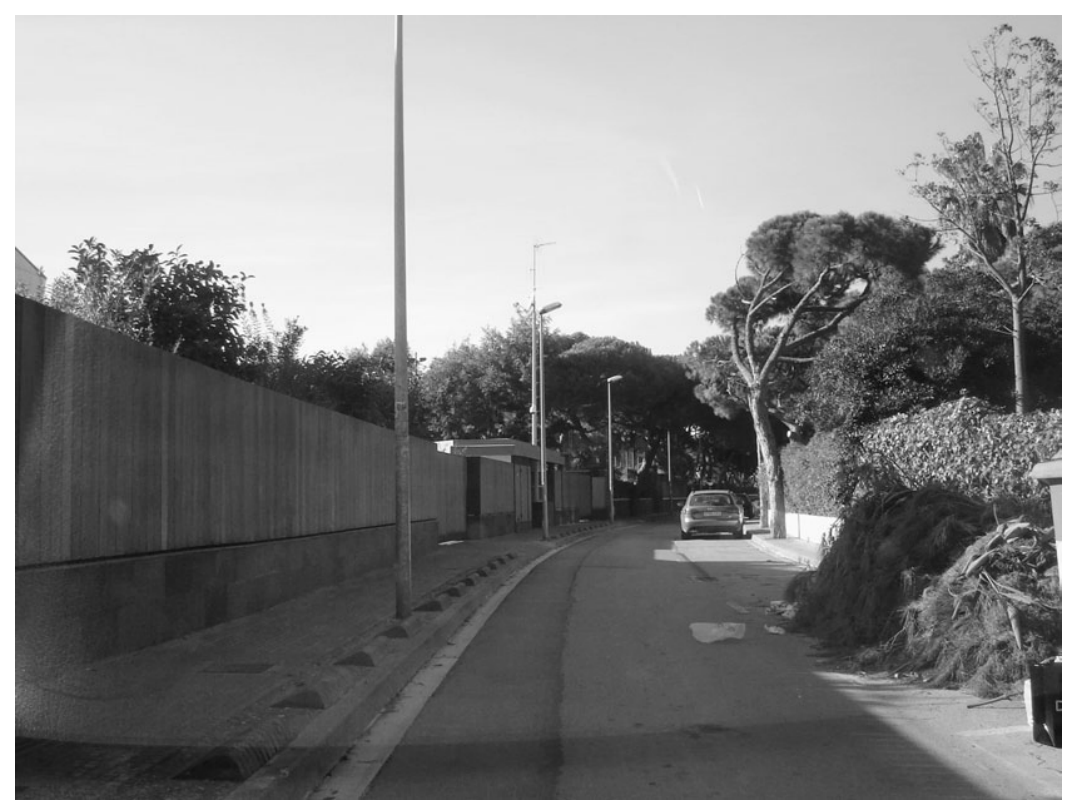

Feminismo/s 17, junio 2011, pp. 105-129 


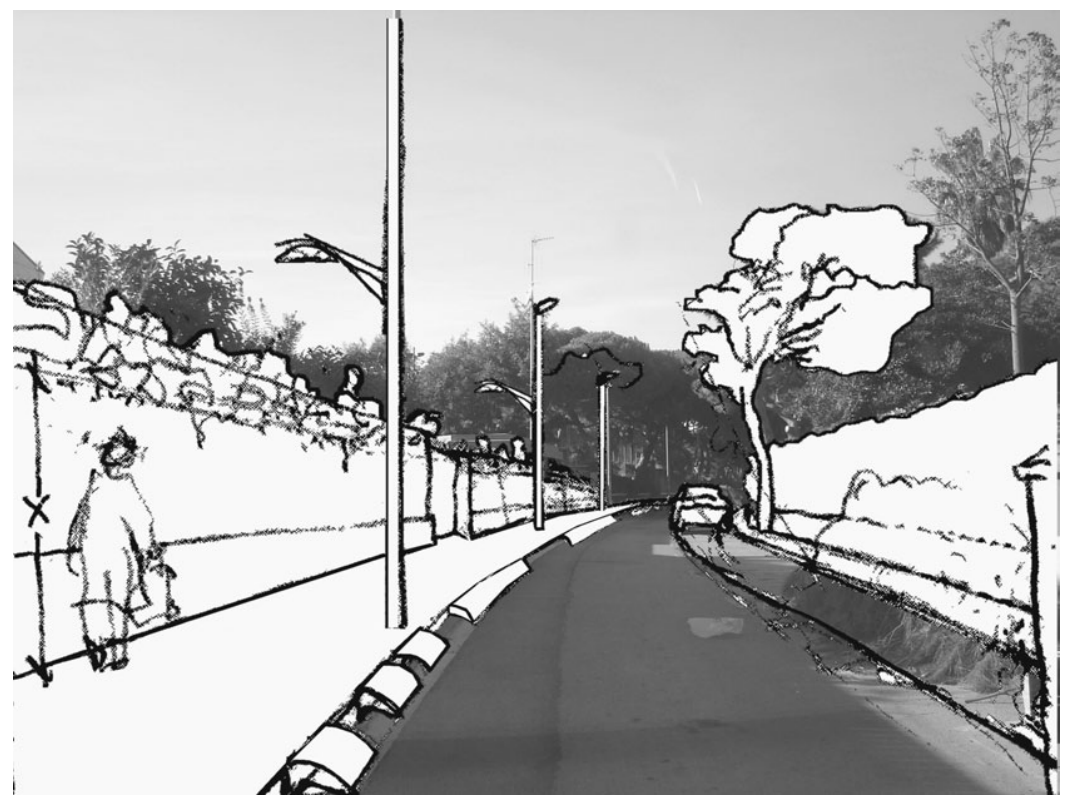

Seguridad

La percepción de seguridad está vinculada a la capacidad de las mujeres de apropiarse del espacio adquiriendo autonomía. Entre los factores espaciales que colaboran en la percepción de seguridad están la visibilidad, la claridad y alternativa de recorridos, la variedad de usos y actividades y la presencia de gente diversa. Para favorecer el sentimiento de pertenencia y seguridad en los espacios públicos se tiene que cuidar especialmente la iluminación de los espacios para peatones y se han de evitar muretes, vallas y escaleras que generen rincones de difícil accesibilidad y escondidos. (Figuras 5 a y 5b)

Todos los estudios sobre seguridad realizados, tanto a nivel local como mundial, nos hablan de una percepción de miedo más importante por parte de las mujeres que de los hombres. Según estudios hechos en Gran Bretaña, la sensación de inseguridad que sufren las mujeres hace que muchas eviten salir por la noche, que modifiquen su recurrido a pie por la ciudad:

(...) estadísticamente las mujeres son generalmente más pequeñas y menos fuertes que los hombres. Las mujeres son las acompañantes de gente mayor o criaturas dependientes el que hace que sus movimientos estén más restringidos (...) las mujeres tienen menos acceso al vehículo privado, y en general cargan con las compras (...) Las circunstancias que hacen que las mujeres tengan miedo a los espacios público en orden de importancia estadística son: la poca luz, la oscuridad, los jóvenes, los hombres sólo, las drogas 

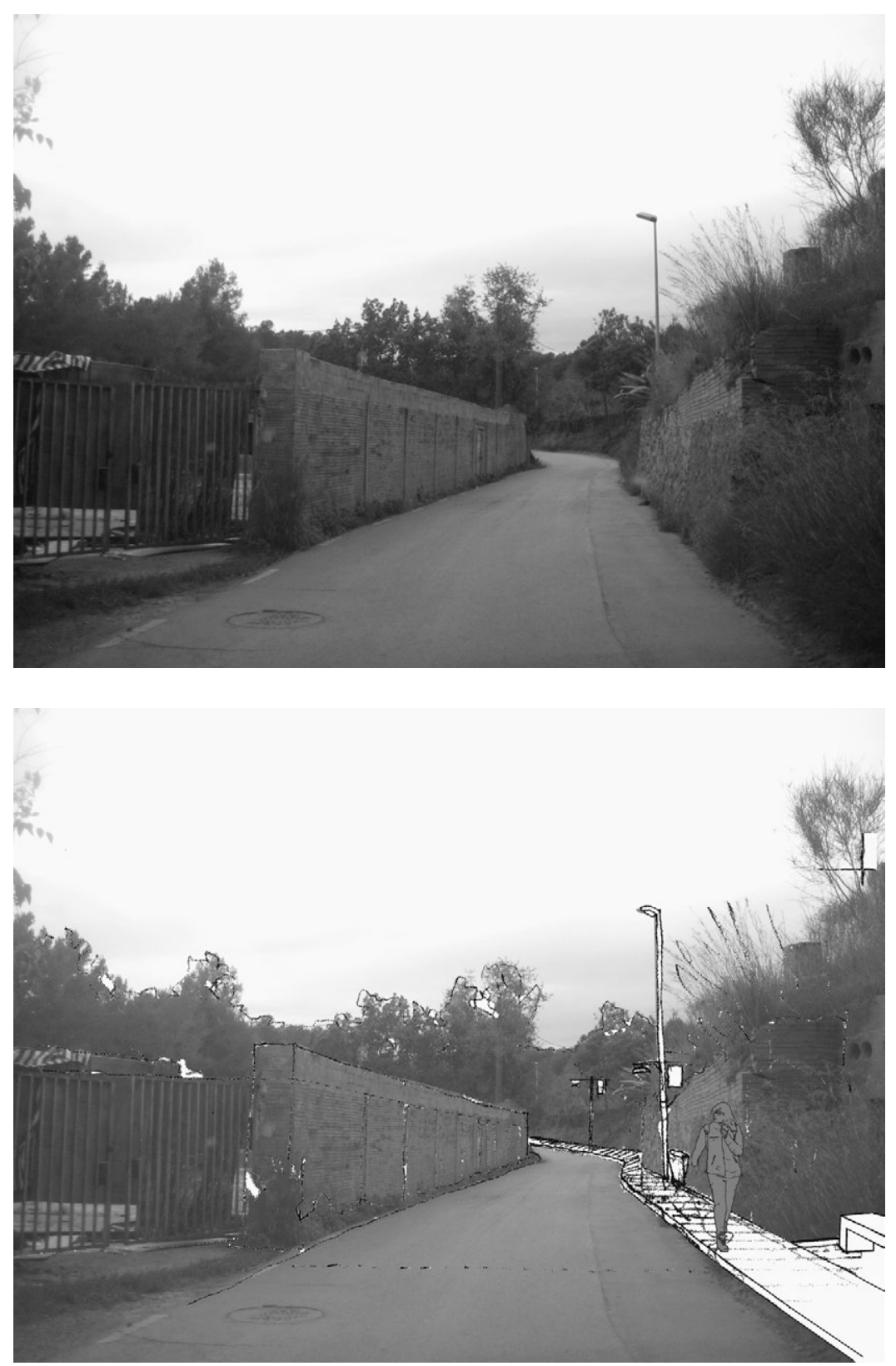

Feminismo/s 17, junio 2011, pp. 105-129 
y el alcohol, el vandalismo, el encontrarse perdida, los perros, y los medios de comunicación $(. . .)^{9}$

\section{Participación}

La participación es imprescindible para comprender y visibilzar lo que ocurre en un espacio, para poner sobre el papel todo el conocimiento que acumula una comunidad sobre su propio territorio y concretamente, la experiencia de las mujeres en su vida cotidiana. Entendemos la participación como un proceso interdisciplinar y transversal, que es necesario que esté presente y sea vinculante desde la diagnosis de la planificación urbanística a la evaluación de la misma.

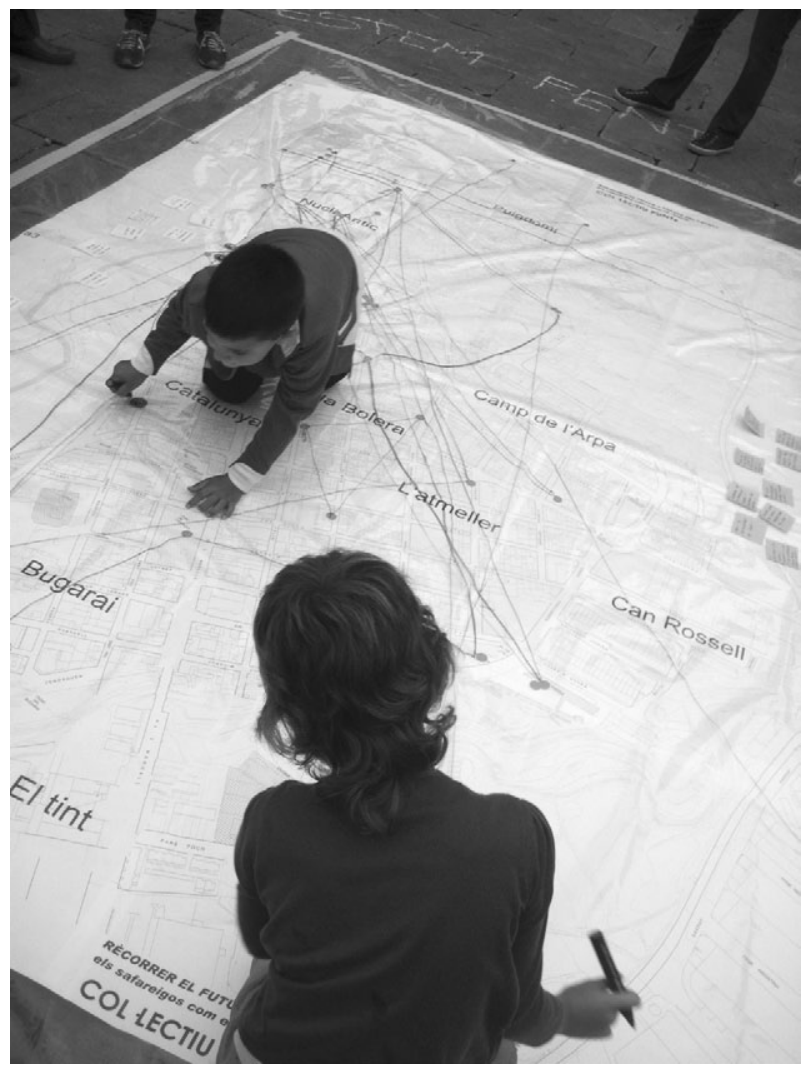

9. Women's Design Service in collaboration with Anne Thorne Architect's Partnership. What to Do About Women's safety in Parks. From A to Y. London: Women's Design Services, 2007, pp. 7, 18-19. 


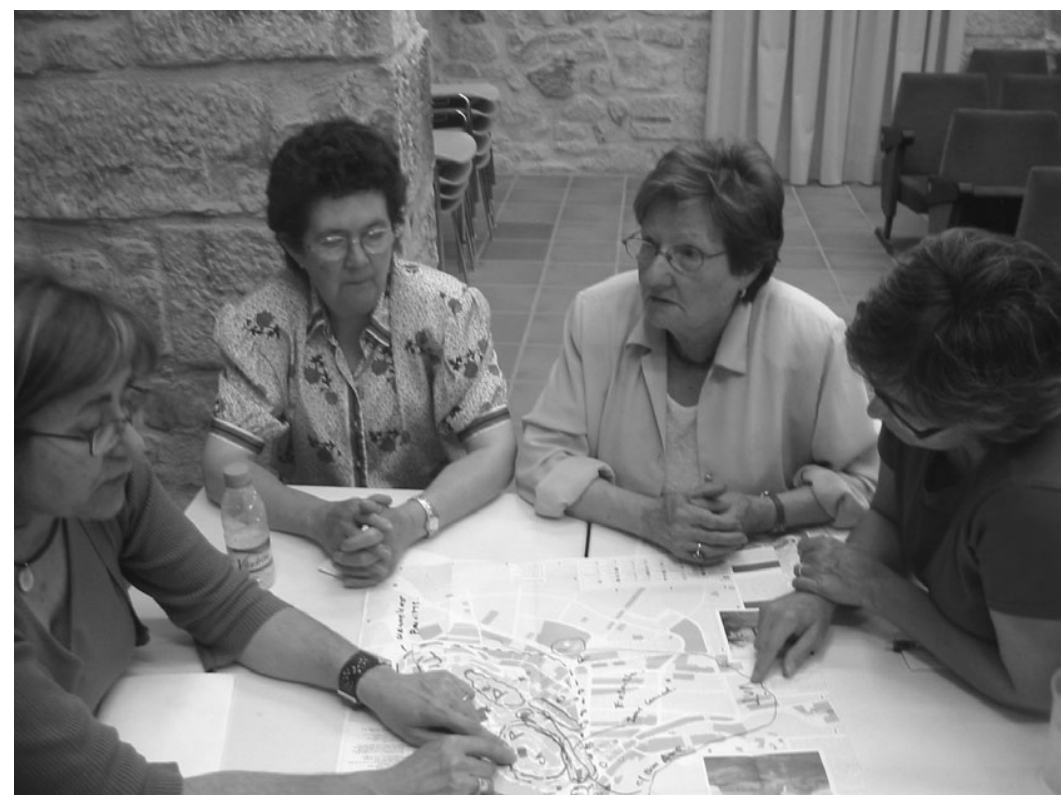

3. Participación desde la experiencia: La experiencia de las mujeres como conocimiento urbano

Entendemos que cualquier intervención en el territorio se tiene que hacer con la participación de las personas que viven, que además serán usuarios y usuarias del espacio resultante. La participación de la ciudadanía en las cuestiones urbanas significa la implicación y la corresponsabilidad en el devenir del entorno que habitan y aumenta el sentimiento de pertenencia al lugar.

La experiencia de las mujeres en la ciudad no ha sido tenida en cuenta como conocimiento dentro de la planificación y el proyecto urbano. El rol del género femenino ha abocado históricamente a las mujeres, y todavía hoy en día a una mayoría, a la realización casi exclusiva de las tareas derivadas del cuidado y atención de los hogares y las personas. Las tareas derivadas del trabajo reproductivo han provocado que las mujeres utilizaran los barrios y las ciudades de forma diferente a la establecida puesto que para los planificadores este conocimiento ha sido invisible o inexistente.

Hoy en día, por ejemplo en Cataluña, las mujeres siguen realizando un $70 \%$ de los trabajos derivados del hogar y la familia, a pesar de que la incorporación al mercado laboral remunerado ha llegado a cotas similares a las masculinas. Estas actividades han dado a la mujer un conocimiento diferencial, 
un conocimiento no tenido en cuenta hasta hoy. Esto lo hemos podido comprobar en los resultados de más de 80 talleres La experiencia de la mujeres en el entorno cotidiano ${ }^{10}$ realizados en los últimos 6 años.

Para poder conocer las experiencias múltiples de las mujeres -múltiples en cada una de ellas y múltiples en la diversidad del grupo- es necesario extraer esta experiencia como dato, como por ejemplo, realizando talleres de participación con los diferentes grupos femeninos que habitan el territorio sobre el cual se trabajará.

Cómo exponen Susan Fainstein y Lisa Servon:

Para la mayor parte de la historia de la planificación urbana las diferencias de género han sido invisibles. (...). La utilización del género como categoría de análisis nos permite deshacer los supuestos que han marcado la teoría y la práctica. Como resultado podemos cuestionar y modificar estos supuestos y alterar la manera que teoría, decisiones y políticas se hacen, para alcanzar y considerar un amplio rango de conocimiento. Para lo cual es necesario hacer diferentes grupos de preguntas, ampliando el rango de métodos que usamos y revaluando el tipo de información que incluimos en el análisis. ${ }^{11}$

No hay duda que los procesos de participación son un paso adelante imprescindible en la democratización de la sociedad. Y es dentro de estos procesos que se tiene que trabajar especialmente con las mujeres. Esta exclusividad está justificada al reconocer la situación de invisibilidad vivida por ellas que les ha traído una falta de seguridad en el autoconocimiento, y, por lo tanto, se hace difícil la participación en igualdad de condiciones cuando el grupo es mixto. Es por este motivo que, a pesar de que en etapas más avanzadas del proceso se reúnen grupos mixtos, es necesario el trabajo en exclusividad sólo con mujeres para generar la confianza necesaria en su conocimiento sobre el hecho urbano.

Si los habitantes de un determinado lugar tienen un saber difícilmente alcanzable exclusivamente desde la observación exterior y los planos, este se hace todavía más evidente cuando son las mujeres quienes participan. Sus experiencias reúnen las de otros grupos, debido precisamente a que son y han sido ellas las encargadas de las personas que necesitan atenciones especiales.

En términos generales un proceso de participación comporta el aprendizaje, la sensibilización y la toma de conciencia crítica para cuestionar el

10. El taller La experiencia de la mujeres en el entorno cotidiano, dinamizado por las autoras de este artículo, forma parte de las Herramientas de participación que el Institut Catalá de les Dones ofrece a las asociaciones y entidades de mujeres en Cataluña desde al año 2005.

11. Fainstein, Susan y Servon, Lisa (ed); Gender and Planning: A Reader. Journal of the American Planning Association, 2007, pp. 1 y 4. 
modelo imperante tanto por parte de la ciudadanía como de técnicos, técnicas, políticos y políticas. Un proceso de participación tiene que tener objetivos estratégicos, transversales y a largo plazo.

\section{Metodologías de análisis urbano desde una perspectiva de género}

Diversos autores y autoras han discutido intensamente durante años sobre la existencia de una metodología propia feminista, sobre si las investigaciones y trabajos que se enmarcan dentro de la teoría feminista utilizan una metodología particular o no. En este artículo no se quiere entrar en este debate, sin embargo, lo que parece evidente es la existencia de unas características específicas cuando se trabaja aplicando la perspectiva de género.

La primera de estas características sería el uso del género como categoría analítica básica, que traspasa el planteamiento teórico del trabajo y se introduce en el diseño metodológico del mismo. Usar la categoría de género obliga a adaptar la metodología de manera que sea capaz de recoger la diversidad de experiencias y necesidades de la población.

La filósofa Sandra Harding, citada por Prats ${ }^{12}$, ha sido una de las autoras que ha tomado parte en estas discusiones, y aunque defiende que no existe un método propio feminista, reconoce las características metodológicas de la investigación sobre género. Por ejemplo, el hecho de que los problemas se plantean desde la perspectiva de la experiencia de las mujeres o el hecho de que se tenga en cuenta el contexto social en el que se realiza la investigación.

Tener en cuenta la experiencia y realidad particular de las mujeres es importante por ejemplo cuando se realizan trabajos participativos, donde para que sea posible la participación en igualdad de condiciones, además de atender las diferentes disponibilidades horarias, tienen que existir facilidades para las personas responsables del cuidado y atención de las personasdependientes.

Otra particularidad de aplicar metodología con perspectiva de género tiene que ver con que la finalidad última de los trabajos va dirigida a influir en el cambio de las relaciones de género. Esta implicación política de los trabajos tiene una repercusión directa en la manera en la que se utiliza la metodología y los resultados que se quieren obtener de ella. Este posicionamiento políticoteórico desde la fase inicial del trabajo, hace que en ocasiones estos trabajos sean tildados de sesgados y poco rigurosos. Estos calificativos podrían entenderse si realmente la ciencia y la praxis pudieran desarrollarse de manera completamente autónoma con respecto las determinaciones sociales, y sin

12. Prats FerRet, María; Geografía feminista i metodología: Reflexió sobre un procès d'aprentatge paral-lel Cuadernos de Geografía no 64, Valencia 1998, pp. 313-323. 
presencia de valores preconcebidos. Aunque, como diversas investigaciones históricas y sociológicas han demostrado, los valores prácticos socialmente dominantes (morales o de otro tipo) acaban repercutiendo en los métodos y los contenidos de la ciencia, bien contribuyendo a seleccionar los objetivos epistémicos que predominan en una determinada fase de la actividad científica, bien suplantando a éstos en el proceso de resolución de los debates científicos. ${ }^{13}$

Por último, otra especifidad del uso de la perspectiva de género está relacionada con la visibilización de la vida cotidiana, independientemente de la escala de aplicación del trabajo. El concepto de vida cotidiana hace referencia a las actividades desarrolladas por las personas en su día a día, indistintamente de la esfera a la que pertenezcan pública y/o privada. Por esto, para evidenciar la complejidad de las prácticas llevadas a cabo en la cotidianeidad, es necesario utilizar una metodología que no invisibilice la realidad cotidiana de parte de la población.

En los trabajamos que hemos desarrollado se han empleado estos puntos como base y además se ha empleado la estrategia de la triangulación múltiple: multidisciplinareidad y diversidad de métodos y datos para desarrollar el trabajo. La diversidad de disciplinas aporta al trabajo variedad de enfoques y puntos de vista. La utilización de distintas técnicas pertenecientes a diversas metodologías (cualitativa, cuantitativa, análisis bibliográfico) aporta al trabajo representatividad y al mismo tiempo información detallada.

A continuación se detallan las metodologías utilizadas generalmente y la manera en la que se aplican.

Primero se hace una aproximación cuantitativa al área de estudio. Se extraen datos estadísticos de diferentes fuentes y posteriormente se analizan. Estos datos son muy útiles para hacer un marco general de la situación, o para trabajar con una escala municipal o metropolitana, sin embargo, cuando se intenta hacer un estudio más pormenorizado, a una escala de barrio o de sección censal, son recurrentes las incoherencias y las deficiencias de los datos, siendo necesario que estos datos vayan acompañados de otro tipo de técnicas que verifiquen y contrasten la información.

A pesar de esto, esta aproximación cuantitativa al área de estudio, ofrece la posibilidad de contextualizar la zona y es un buen punto de partida para poder implementar el resto de técnicas de investigación.

13. RODRIgUeZ AlCAZAR, Javier; Esencialismo y neutralidad científicas en el libro Ciencia, Tecnología y Sociedad. Contribuciones para una cultura de la paz. Ed. Rodríguez Alcázar, Medina Doménech y Sánchez Cazorla, Ed. 1997, pp. 49-84. 
La utilización de herramientas cualitativas en los análisis urbanos nos permite incorporar datos no cuantificables dentro de los datos estadísticos y trabajar a una escala de detalle para la que no existen datos. Para desarrollar la parte cualitativa de la investigación se emplean diferentes técnicas que intentan dar respuesta a una serie de cuestiones básicas: quién usa o quién vive, con quién, por qué, y cómo. ${ }^{14}$ La primera es la observación participante. Esta técnica permite la verificación de los datos cuantitativos así como obtener información primaria de fenómenos difícilmente cuantificables en estadísticas. Está compuesta por una serie de técnicas de obtención y análisis de datos y permite aproximarse a la realidad social intentando observarla de modo directo, entero y en su complejidad, sin artificios ni simplificaciones y en el momento en que sucede el fenómeno que se quiere estudiar. Posibilita una visión total de la realidad, teniendo en cuenta el contexto y sin fragmentar lo real. Ningún fenómeno social puede ser entendido fuera de sus referencias espacio-temporales y de su contexto. ${ }^{15}$

La observación consta de un análisis secuencial en distintos espacios para cada uno de los contextos seleccionados a diferentes horas del día (mañana, tarde, noche) y en días distintos (laborable, sábado, festivo)

Para recoger la información de las observaciones se utilizan una serie de fichas (Col-lectiu punt $6^{16}$ ) que clasifican la información según dos dimensiones (física y social) lo que facilita su posterior análisis. La utilización de las fichas de recogida de datos, sistematiza la información lo que permite comparar diferentes contextos espaciales y temporales.

Durante las observaciones se hacen entrevistas espontáneas a personas usuarias de los espacios públicos. Estas entrevistas no son estructuradas y se pretende obtener información de primera mano acerca de las percepciones que tienen las personas de los espacios.

Por último, se realizan entrevistas en profundidad que aportan información adicional a la conseguida mediante las observaciones y las entrevistas espontáneas, además de corroborar las dinámicas observadas

Durante la realización del trabajo de campo se toman fotografías y se realizan diagramas y dibujos para complementar la información obtenida y como medio para depurarla.

14. RAINERO, Liliana y RODIGOU, Maite; Indicadores urbanos de género. Instrumentos para la gobernabilidad urbana. Córdoba Argentina CICSA, 2001.

15. VAlLes, Miguel; Técnicas cualitativas de investigación social. Reflexión metodológica y práctica profesional. Madrid Editorial Síntesis, 1999.

16. MuXí MARTínEZ, Zaida y COL·LECTIU PUNT 6; 2006-2007. 


\section{Conclusiones}

Por todo lo expuesto entendemos que una sociedad que aspire a estar formada por seres humanos en igualdad, sin factores discriminatorios por sexo, clase, capacidades físicas, raza o edad, tiene que planificar sus ciudades -incluyendo todas las escalas y todos los ámbitos- desde la visibilidad y la igualdad en importancia de las dos esferas en las cuales se han dividido los trabajos de las personas tradicionalmente. Es imprescindible reconocer que la manera como se construyen las ciudades no es neutral. Afectará de diferente manera a las personas si no se han tenido en cuenta las heterogéneas necesidades de la población, poniendo en primer plano los requerimientos del trabajo reproductivo. Por eso, pensar el espacio urbano para todos y para todas es hacerlo desde la diferencia pero no desde la desigualdad: una diferencia entre hombres y mujeres, entre clases, entre orígenes, culturas, religiones, etc. posicionando en igualdad de condiciones todas las demandas, sin decisiones a priori, que excluyan la experiencia de las personas como fuente fundamental de conocimiento en las deciciones urbanas.

\section{Referencias bibliográficas}

AAVV; La Carta Europea de la Mujer en la Ciudad, Bruselas (Bèlgica). Experiència seleccionada en el Concurso de Buenas Prácticas patrocinado por Dubai en 1996.

AAVV; Urbanisme i gènere. Una visió necessària per a tothom. Barcelona, DIBA, Xarxa de Municipis, 2006.

AAVV; Manual práctico para una señalización urbana igualitaria. Sexismo en la señalización. FEMP, Federación Española de Municipios y Provincia Ministerio de Igualdad.

BOFILL LEVI, Anna; Guia per al planejament urbanístic i l'ordenació urbanística amb la incorporació de criteris de gènere. Col•lecció Eines $n^{\circ} 11$. Barcelona, Generalitat de Catalunya, Institut Catalá de les Dones, Departament de Política Territorial i Obres Públiques, 2008.

Bofill LeVI, Anna; Planejament urbanístic, espais urbans i espais interiors des de la perspectiva de les dones. Quaderns de l'Institut / 6. Barcelona, Generalitat de Catalunya, Institut Catalá de les Dones, Departament de Política Territorial i Obres Públiques, 2005.

BоотH, Christine; «La participación de las mujeres en la planificación y el desarrollo urbanístico», en las jornadas del País Vascó organizado por la Diputación Foral de Bizkaia, gabinete del Diputado General. 2005. 
Carrasco, Cristina i Serrano, Mònica; Compte satèl $\bullet$ lit de la producció domèstica (CSPD) de les llars de Catalunya 2001, col•lecció Estudi 1, Institut Català de les Dones, Generalitat de Catalunya, octubre del 2006.

CRUELls, Eva y PARICIO, Anna; La ciutat possible i desitjada per les dones: recercaper un diseny i planifi cació urbanística des d'una perspectiva de gènere. Investigación para la Diputación de Barcelona, Barcelona, 2007.

FAinstein, Susan y Servon, Lisa (ed); Gender and Planning: A Reader. Journal of the AmericanPlanning Association, 2007.

GREED, Clara; «Genero y planificación del territorio. ¿Un mismo tema?» en el Fórum internacional de planificación del territorio desde una perpectiva de género, organizado por la Fundació Aurèlia Campany, 17 de noviembre de 1997.

JACOBS, Jane; Muerte y Vida de las grandes ciudades. Barcelona: Ed. 62, 1967.

LIENAS, Gemma; El diari lila de la Carlota. Barcelona: Editorial Empuries, 2001.

Miralles-Guasch, Carme; Dones, mobilitat, temps i ciutats, col-lecció Quaderns de l'Institut 14, perspectives des del feminisme, Institut Català de les Dones, Barcelona, juny 2010.

MuXí MARTINEZ, Zaida y COL.LECTIU PUNT 6; Recomanacions per la implementació de expediente U-62/06. Convenio de colaboración con la Universitat Politècnica de Catalunya grupo de investigación I-00868. 2006-2007.<www. punt6.net>.

Muxí MARTíneZ, Zaida; de «Barris per a viure» en Nous Horintzons. Els barris invisibles $n^{\circ} 195$, editada per la Fundació Nous Horitzons, Barcelona, 2009.

MUXí MARTíNEZ, Zaida; de «Infraestructures socials i de proximitat, equipaments per a la vida quotidiana» en la revista Quaderns d'Acció Social i Ciutadania. Revista d’informació, anàlisi i investigació socials. Generalitat de Catalunya. Departament d'Acció Social i Ciutadania, n³. Barcelona (2008) pp. 60-62.

Muxí Martínez, Zaida; de «Ciudad Pròxima. Urbanismo sin Género.» a la Revista Ingeniería y Territorio $n^{\circ} 75,2006$. Revista del Colegio de Ingenieros de Caminos, Canales y Puertos, Barcelona.

Muxí MARTíneZ, Zaida; «Decir la ciudad desde la experiencia» en la revista Arquitectos, Construcción de la ciudad, $n^{\circ} 2$, 2006. Revista de información del Consejo Superior de los Colegios de Arquitectos de España. Madrid.

Muxí MARTíneZ, Zaida; de «El espacio no es neutro: reflexiones en torno al derecho a la vivienda y la ciudad desde una perspectiva de género» en Boletín Derecho por el derecho a la vivienda y contra los desalojos. Diciembre 2008 - enero 2009.

MuXí MARTínEZ, Zaida; Urbanisme i Génere. El paper de les Dones en la configuració de la ciutat. De Dossier en Femení del programa municipal de la Dona de l'ajuntament de l'Hospitalet, Hospitalet del Llobregat, 2008. 
Muxí MartíneZ, Zaida; «Ciutat Próxima. Una crítica feminista a la ciutat global» en el llibre Sota les Llambordes, la platja, de José Miguel G.Cortés, editen H. Associació per a les Arts Contemporánies y Eumo editorial, Vic, Barcelona.

PRATS FERRET, María; Geografía feminista i metodología: Reflexió sobre un procès d'aprentatge paral.lel Cuadernos de Geografia no 64, pag 313-323, Valencia 1998.

RAINERO, Liliana y RODIGOU, Maite; Indicadores urbanos de género. Instrumentos para la gobernabilidad urbana. Córdoba Argentina CICSA, 2001.

RODRIGUEZ AlCAZAR, Javier; Esencialismo y neutralidad científicas en el libro Ciencia, Tecnología y Sociedad. Contribuciones para una cultura de la paz (ed: Rodríguez Alcázar, Medina Doménech y Sánchez Cazorla) pp. 49-84, 1997.

SÁNCHEZ DE MADARIAGA, Inés; Urbanismo con prespectiva de género, Ed. Junta de Andalucía, Instituto Andaluz de la Mujer.

SOLSONA, Glòria; Introducció a la perspectiva de gènere en els procesos de participacióciutadana. Diputació de Barcelona, Barcelona, 2007.

VAlles, Miguel; Técnicas cualitativas de investigación social. Reflexión metodológica y práctica profesional. Madrid Editorial Síntesis, 1999.

VelázQuez, Isabela, Gea 21; Instrumentos, métodos y técnicas para la participación. Presentación Flor de Maig 2005.

WOMEN'S DESIGN SERVICE en colaboración con ANNE THORNE ARCHITECT'S PARTNETSHIP; What to Do About Women's safety in Parks. From A to Y. London: Women's Design Services, 2007.

Pàginas webs:

www.punt6.net

www.diba.cat/dretshumans

www.gencat.cat/icdona/

www.wds.org.uk/

www.habitat.aq.upm.es/boletin/n7

www.generourban.org

www.lamujerconstruye.org

www.redmujer.org.ar

www.mujeresyciudades.org 